\title{
Consciousness of targets during the attentional blink: a gradual or all-or-none dimension?
}

\author{
Sander Nieuwenhuis • Roy de Kleijn
}

Published online: 6 November 2010

(C) The Author(s) 2010. This article is published with open access at Springerlink.com

\begin{abstract}
Models of consciousness differ in whether they predict a gradual change or a discontinuous transition between nonconscious and conscious perception. Sergent and Dehaene (Psychological Science, 15, 720-728, 2004) asked subjects to rate on a continuous scale the subjective visibility of target words presented during an attentional blink. They found that these words were either detected as well as targets outside the attentional-blink period or not detected at all, and interpreted these results as support for a discontinuous transition between nonconscious and conscious processing. We present results from 4 attentionalblink experiments showing that this all-or-none rating pattern disappears with the use of an alternative measure of consciousness (post-decision wagering) and a more difficult identification task. Instead, under these circumstances, subjects used the consciousness rating scales in a continuous fashion. These results are more consistent with models that assume a gradual change between nonconscious and conscious perception during the attentional blink.
\end{abstract}

Keywords Attentional blink - Consciousness · Postdecision wagering $\cdot$ Awareness $\cdot$ Subjective ratings

Electronic supplementary material The online version of this article (doi:10.3758/s13414-010-0026-1) contains supplementary material, which is available to authorized users.

S. Nieuwenhuis $(\bowtie) \cdot R$. de Kleijn

Institute of Psychology, Cognitive Psychology Unit,

Leiden University,

Wassenaarseweg 52 ,

2333 AK Leiden, The Netherlands

e-mail: snieuwenhuis@fsw.leidenuniv.nl

\section{Introduction}

An important question in research on consciousness is whether there is a continuous or discontinuous transition between nonconscious and conscious perception. In support of the former, imaging studies of visual perception have found gradual increases in the strength of cortical activity as subjective perception of a stimulus improved (e.g., Moutoussis \& Zeki, 2002). According to this view, conscious perception is achieved when the strength of a perceptual representation crosses a threshold toward the higher end of a continuum of (un)conscious states. Other studies have reported all-or-none differences in neural activation between nonconscious and conscious perception of a stimulus (Dehaene et al., 2001; Lamme, Supèr, Landman, Roelfsema, \& Spekreijse, 2000), suggesting a discontinuous transition between the two states. Such qualitative differences between nonconscious and conscious processing are often predicted by models that consider recurrent interactions between distant brain areas a prerequisite for conscious perception (Dehaene, Sergent, \& Changeux, 2003; Lamme, 2003). For example, the global neuronal workspace model (Dehaene et al., 2003) assumes that bottom-up neural activity may be sufficiently strong to trigger self-amplifying recurrent activity, resulting in conscious perception. Progressively decreasing the strength of this bottom-up activity initially leads to a gradual decrease in global activation and thus in subjective visibility. At a certain threshold, however, there is a discontinuous jump to a lower level of activation, corresponding to a lack of sustained activation and therefore an absence of conscious perception.

Sergent and Dehaene (2004) have tested contrasting predictions of discontinuous- and continuous-transition models by examining subjective visibility ratings of participants in an attentional-blink experiment (reviewed in Martens \& Wyble, 2010). In each trial, participants were 
presented with two targets embedded in a rapid serial visual presentation (RSVP) stream of consonant strings. Participants had to identify the first target (T1: OXXO or XOOX) and rate the subjective visibility of the second target (T2: the French number word 'DEUX, 'CINQ', 'SEPT' or 'HUIT'), which was present in only half the trials. Subjective visibility ratings were made on a 21-point horizontal scale labeled 'not seen' on the left end and 'maximal visibility' on the right end. The number of items (lag) between $\mathrm{T} 1$ and $\mathrm{T} 2$ was systematically varied between 1, 2, 3, 4, 6 and 8. As expected, mean T2 visibility ratings in T2-present trials were high when T2 was presented outside the attentional-blink period (i.e., at lags 6 and 8), and strongly reduced when $\mathrm{T} 2$ was presented during the attentional-blink period (at lags 2-4).

The critical predictions concerned the distributions of $\mathrm{T} 2$ visibility ratings in T2-present trials. Sergent and Dehaene (2004) argued that a model postulating a continuum of perception would predict that the attentional blink is characterized by a gradual degradation of the T2 word representations. This should result in a gradual displacement of subjective ratings toward lower subjective degrees of visibility. In contrast, discontinuous-transition models like the global neuronal workspace model would predict that subjective visibility ratings show a bimodal distribution, and that the attentional blink is characterized by an increase of one peak (indicating that $\mathrm{T} 2$ was not seen) at the expense of the second peak (indicating that T2 was seen). The subjective visibility ratings reported by Sergent and Dehaene showed a striking pattern consistent with a discontinuous transition between nonconscious and conscious perception of T2. Participants used the visibility scale in an all-or-none fashion: targets were either identified as well as targets presented outside the attentional-blink period or not detected at all. Changes across lags in the strength of the attentional blink were characterized by changing proportions of 'seen' and 'not seen' ratings at both ends of the subjective visibility scale. Sergent and Dehaene interpreted these findings as support for a sharp nonlinear transition between nonconscious and conscious perception.

In this study, we addressed three potential limitations of Sergent and Dehaene's (2004) experiment. The first potential limitation concerns the nature of the subjective visibility scale used. As previously noted by others (Overgaard, Rote, Mouridsen, \& Ramsøy, 2006), it is unlikely that people are able to recognize or report such fine gradations of subjective visibility as to justify the use of a 21-point scale. Furthermore, the fact that only the end positions on the scale were labeled may have made it more difficult for participants to use intermediate (unlabeled) positions. Therefore, the visibility ratings in Sergent and Dehaene's experiment may have been all-or-none because only the two extreme ends of the scale were labeled and the intermediate points on the scale did not have sufficient meaning to the participants. In Experiment 1, we addressed this possibility by examining whether we could replicate Sergent and Dehaene's findings using a 7-point scale.

The second potential limitation concerns the use of subjective visibility ratings as a measure of consciousness. It has been noted that subjective reports such as those used in Sergent and Dehaene (2004) may not have sufficient 'null sensitivity'. That is, participants tend to withhold minimal conscious knowledge of weakly perceived stimuli (cf. Overgaard et al., 2006). Persaud, McLeod, and Cowey (2007) have recently proposed that this limitation is resolved by an alternative method called post-decision wagering. Participants are required to first make a psychophysical decision and then place a bet on its correctness by making a higher or lower wager. If the decision is correct, the participant wins the amount of money wagered; if it is incorrect, the participant loses that amount. Participants thus have the opportunity to gain money on the basis of their psychophysical performance. Researchers have argued that post-decision wagering is a more implicit way to assess awareness, and a more intuitive measure for participants than introspection (Koch \& Preuschoff, 2007; Persaud et al., 2007; but for a critique see Clifford, Arabzadeh, \& Harris, 2007). In Experiment 2, we examined whether Sergent and Dehaene's findings would generalize to an experiment that replicated their attentional-blink task but used post-decision wagering instead of subjective visibility ratings.

A third peculiar aspect of the study by Sergent and Dehaene (2004) is that the T2 stimuli, of which participants had to rate the subjective visibility, were words. Even if word recognition is initially disrupted by a pattern mask (e.g., the $\mathrm{T} 2+1$ distractor), our cognitive system is often able to use perception of single letters to complete the representation of the word, based on interactive activation between representations at the letter level and word level (McClelland \& Rumelhart, 1981). This mechanism may considerably ameliorate the attentional blink. In Experiments 3 and 4, the distractors and targets in the RSVP stream were single characters, as is typical in attentionalblink research, and participants had to identify T2 (which was present in half the trials) as well as rate its subjective visibility. For what it is worth, informal reports (in exit interviews) from participants in previous studies using this stimulus material suggest graded levels of consciousness of the targets - an experience that is apparently inconsistent with the all-or-none perception reported by Sergent and Dehaene. In Experiment 3, we combined the characteridentification task with subjective visibility ratings, and in Experiment 4 with post-decision wagering. 


\section{Experiment 1}

Experiment 1 was a replication of Sergent and Dehaene's (2004) first experiment, with two notable exceptions. We did not include a single-task condition. More importantly, we used a 7-point instead of 21-point scale for indicating subjective visibility of $\mathrm{T} 2$.

\section{Method}

Participants Twelve students of Leiden University participated in a session of approximately $45 \mathrm{~min}$ in return for $€ 6.50$.

Stimuli, procedure, and design Stimuli were presented in blue (cyan) on a black background, except for T1 which was presented in white. Each trial started with a fixation cross, presented for $1,000 \mathrm{~ms}$ at the center of the computer screen. Then, the fixation cross was replaced by an RSVP stream of 10 to 20 letter strings, each measuring $4^{\circ} \times 1^{\circ}$. All items were displayed for $43 \mathrm{~ms}$, separated by 71-ms blanks. T1 could either be "OXXO" or "XOOX", while T2 was a Dutch four-letter number word, "DRIE", "VIER", "VIJF", or "ACHT", similar to the French number words used by Sergent and Dehaene (2004). All other items were distractors, consisting of four random consonants. T1 was presented at positions 7 or 10 in the stream. The temporal distance between $\mathrm{T} 1$ and $\mathrm{T} 2$ was systematically varied between 1, 2, 3, 4, 6 and 8 items, corresponding to lags of 114, 228, 342, 456, 684, and $912 \mathrm{~ms}$. After T2, two more distractors were displayed.

Immediately after the RSVP stream, participants were asked to rate the subjective visibility of T2 ("... indicate how well you have seen the second target ...") on a 7-point horizontal scale, labeled "not seen" at the left and "maximal visibility" at the right end. Participants moved a cursor on the scale by pressing the left and right arrow keys on a keyboard, and then validated their choice by pressing the space bar. To prevent response bias, the initial cursor position was randomly determined for each trial. Subsequently, participants were asked to identify the two middle letters of T1 (either "OO" or "XX") by pressing the O or X key.

In half the trials, T2 was replaced with a blank screen. Also, to prevent $\mathrm{T} 2$-absent trials from being too obvious, $20 \%$ of all distractors were replaced with a blank screen, except for distractors immediately preceding or following $\mathrm{T} 1$ or $\mathrm{T} 2$, and the last distractor in the RSVP stream. Totals of 32 trials for each lag were run for both the T2-present and T2-absent conditions, resulting in a total of 192 T2-present trials and 192 T2absent trials.
Results and discussion

One participant who rated "not seen" in all trials was discarded from further analyses. Trials with an incorrect response to $\mathrm{T} 1(M=14.6 \%)$ were discarded. A GreenhouseGeisser correction was applied where appropriate. An analysis of variance showed a significant effect of lag on mean visibility ratings in T2-present trials, $F(5,50)=$ $7.0, p=.009$. Mean visibility ratings dropped from $77 \%$ at lag 1 to $65 \%$ at lag 2 , then increased to $83 \%$ at lag 6 , indicating a typical attentional blink (Fig. 1). In T2-absent trials, mean visibility ratings were very low and unaffected by lag.

Figure 2 shows the rating distributions in T2-absent and T2-present trials as a function of lag. In the T2-absent trials, the distributions showed a single peak at $0 \%$ visibility ( $>80 \%$ of the trials). In contrast, in T2-present trials in which T2 was clearly visible (outside the attentional blink, at lags 6 and 8), the distribution showed a prominent peak at $100 \%$ visibility ( $\sim 55 \%$ of the trials). Importantly, the visibility ratings for T2-present trials during the attentional-blink period showed a clearly bimodal pattern: in some trials, participants gave high visibility ratings, similar to those obtained outside the attentional-blink period, while in the other trials they used the lowest visibility rating ( $\sim 15 \%$ of the responses at $0 \%$ visibility), indicating an attentional blink. Some of the intermediate ratings were rarely chosen (especially 17 and 33\%). However, the 67 and $83 \%$ ratings were chosen more often, suggesting that perception of T2 was not all-or-none as in Sergent and Dehaene (2004). Indeed, the pattern of visibility ratings was more similar to that obtained in a replication study (including EEG) by Sergent, Baillet, and Dehaene (2005), who abandoned the term "all-or-none" and instead referred to a "discontinuous transition" between nonconscious and conscious perception.

Following Sergent and Dehaene (2004), we tested the hypothesis that ratings during the attentional blink reflect a mixture of discrete seen and not-seen states by submitting the grand-average rating distributions for the T2-present trials (lags 1-6) to a linear regression, using two predictors: the rating distribution observed when $\mathrm{T} 2$ was present at lag 8 (present predictor), and the distribution observed when T2 was absent at lag 8 (absent predictor). The present predictor explained a significant portion of the variance for all lags $(p s<.002)$. The contribution of the absent predictor was significant for lag $3(p=.037)$, marginally significant for lag $2(\mathrm{p}=.077)$, and not significant for the other lags. The contribution of the absent predictor for lags 2 and 3 suggests that during the attentional blink some of the T2-present trials were subjectively indistinguishable from T2-absent trials. 
Fig. 1 Left Mean subjective visibility of $\mathrm{T} 2$ as a function of $\mathrm{T} 1-\mathrm{T} 2$ lag and $\mathrm{T} 2$ presence in Experiments 1 and 3. Right Mean wagers on the presence or absence of $\mathrm{T} 2$ as a function of $\mathrm{T} 1-\mathrm{T} 2$ lag and factual T2 presence in Experiments 2 and 4

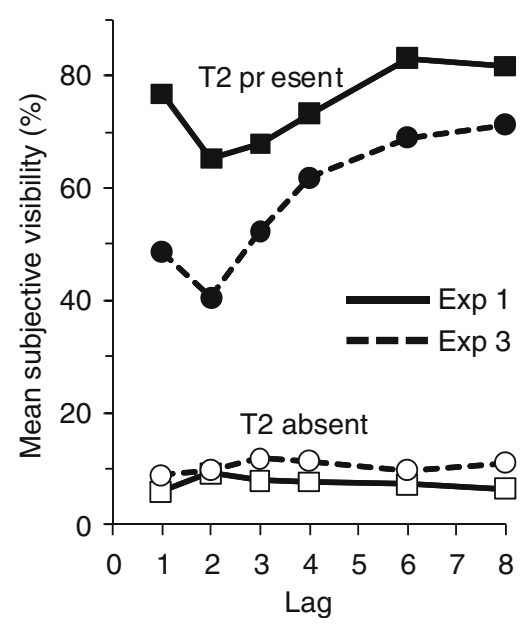

To further compare our results with those of Sergent and Dehaene (2004, Experiment 1), we re-grouped their 21-bin data into 7 bins by grouping together the observations from each 3 original bins. The resulting visibility distributions are shown in Fig. 3, alongside our T2-present distributions. Although we cannot infer that Sergent and Dehaene's data would have looked like this had they used 7 instead of 21 bins, the similarity between the distributions from the two experiments is striking.

Thus, despite the use of a 7-point rating scale, we replicated the bimodal rating pattern reported by Sergent and Dehaene (2004) and, to some extent, the all-or-none character of that pattern, although "discontinuous transition" is perhaps a safer description. We emphasize that 7 is still a rather arbitrary choice of scale points. Other authors have advocated the use of a 4-point scale (the Perceptual Awareness Scale; Ramsøy \& Overgaard, 2004) and have supported the strength of this scale with empirical data (e.g., Sandberg, Timmermans, Overgaard, $\&$ Cleeremans, in press).

\section{Experiment 2}

In Experiment 2, we replicated the previous experiment, but using post-decision wagering as an alternative measure of awareness.

\section{Method}

All methods were identical to Experiment 1, except when noted below. Thirteen participants were paid $€ 6.50$ plus a performance-related bonus, as described below. Each RSVP sequence was followed by the presentation of a scale almost identical to the visibility scale used in Experiment 1. However, instead of judging T2 visibility, participants were asked to wager an amount of money on the presence or absence of T2 (“... indicate how much money you are prepared to wager on the presence or absence of the second target."). To optimize the comparison with the visibility scale in Experiment 1, we used a post-decision wagering scale that collapsed the decisions about presence/absence and wagered amount into a single decision. This contrasts with previous research in which participants were asked to make these decisions sequentially (e.g., Persaud et al., 2007). The scale consisted of seven positions: $€ 0.60, € 0.40$, and $€ 0.20$ wagers on the absence of $\mathrm{T} 2$, a $€ 0$ wager, and $€ 0.20, € 0.40$, and $€ 0.60$ wagers on the presence of $\mathrm{T} 2$. The scale was labeled "absent" at the left end and "present" at the right end. When participants rated T2 correctly as absent or present (i.e., when they wagered an amount on the correct side of the horizontal wagering scale), they received the amount of money wagered. When they wagered an amount on the wrong side of the wagering scale, they lost the amount wagered. Participants were informed that a randomly determined $10 \%$ of all post-decision wagers would be paid after the experiment.

Results and discussion

Trials with an incorrect $\mathrm{T} 1$ identification were discarded $(M=13.0 \%)$. For $\mathrm{T} 2$-present trials, mean wager scores dropped from $€ 0.49$ (lag 1) to $€ 0.41$ (lag 2), then increased to $€ 0.49$ at lag 6 (Fig. 1). Although this trend follows the typical attentional-blink pattern, the effect of lag was nonsignificant, $F(5,60)=2.7, p=.10$, indicating that participants did not show a reliable attentional blink.

The wager distributions, plotted in Fig. 2, show a striking pattern. In the T2-absent trials, participants generally wagered ' $\mathrm{T} 2$ absent', although the wager scores were more distributed than the visibility ratings in Experiment 1. Interestingly, the distributions for T2-present trials showed 
T2 absent

T2 present
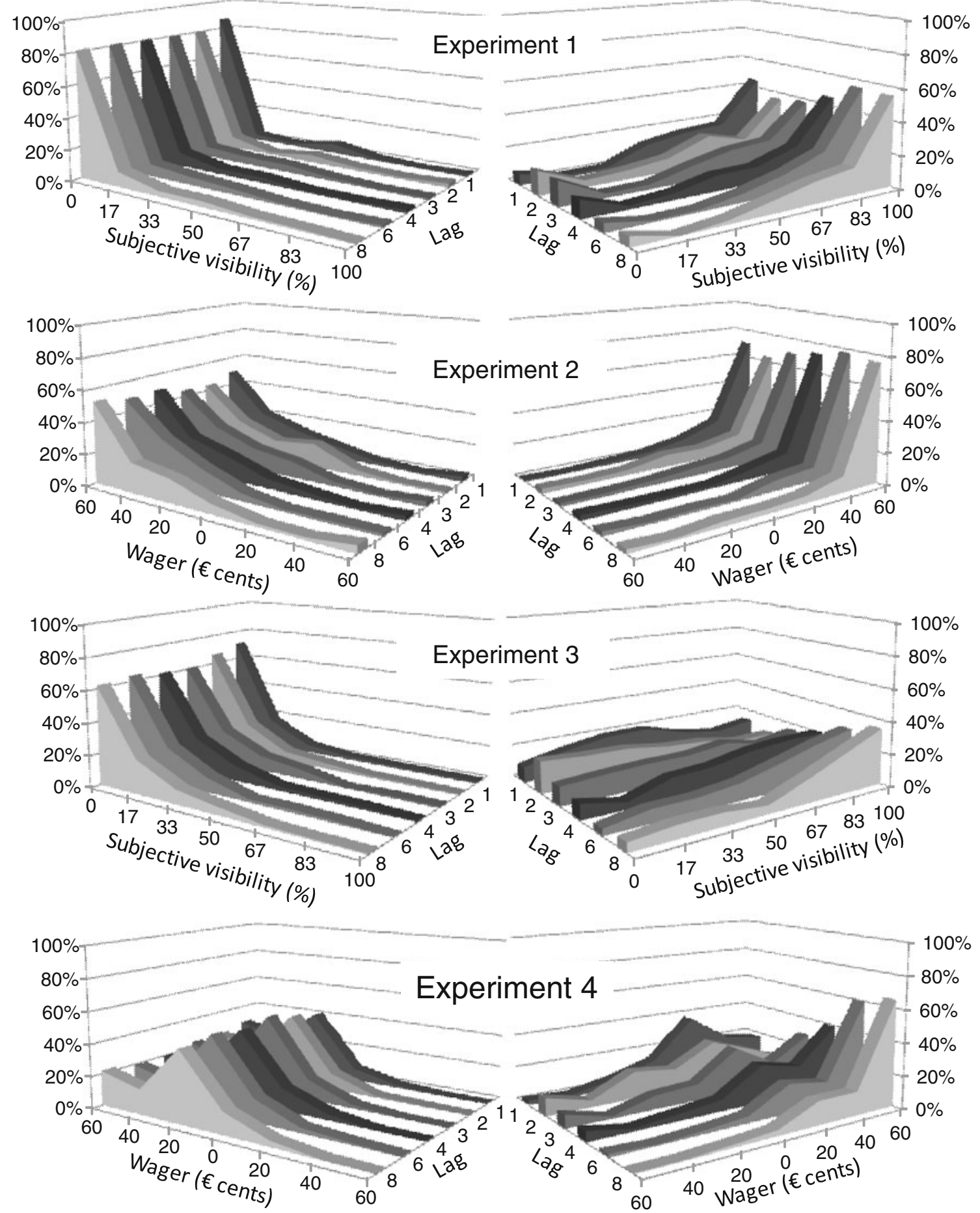

Fig. 2 Response distributions on T2-absent (left) and T2-present trials (right) as a function of T1-T2 lag for each of the four experiments. The ordinates indicate percentage of trials. The numbers plotted in these graphs are reported in Supplementary Table 1

a single, large peak at the maximum T2-present wager ( $>63 \%$ of the trials), and hardly any $\mathrm{T} 2$-absent wagers $(<9 \%$ wagers to the left of $€ 0)$, even during the attentional blink - a unimodal pattern very different from the bimodal pattern reported in Sergent and Dehaene (2004) and
Experiment 1 . This was confirmed by a linear regression analysis of the grand-average wager distributions for T2present trials (lags 1-6), which indicated significant contributions of the present predictor $(p s<.001)$ but not of the absent predictor. 
Sergent \& Dehaene (2004)

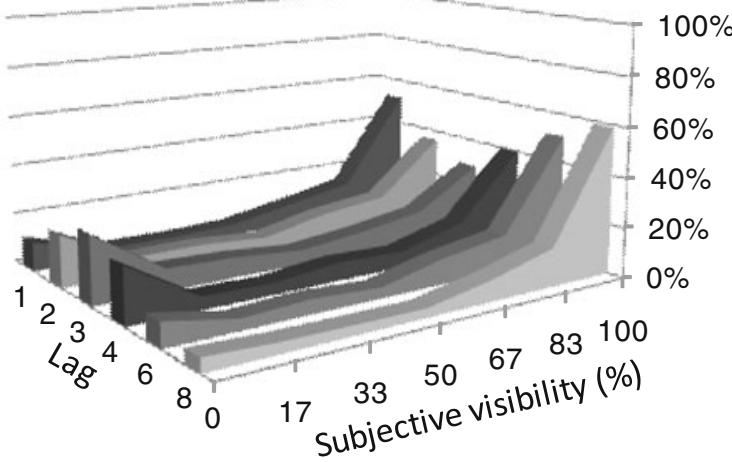

Experiment 1

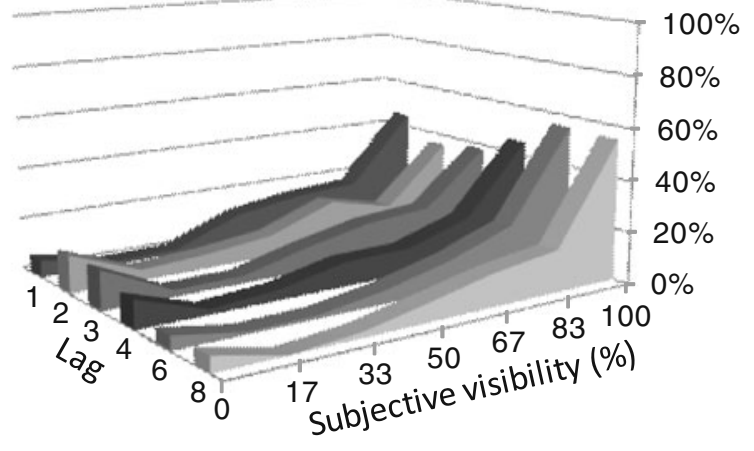

Fig. 3 Response distributions on T2-present trials as a function of T1-T2 lag for the re-grouped data of Sergent and Dehaene (2004, Experiment 1) and Experiment 1. The ordinates indicate percentage of trials

What does this wager pattern mean? One possible explanation is that the monetary reward associated with good performance increased the participants' effort or motivation and that this led to an increased perceptual sensitivity (i.e., $d$ ' in signal-detection theory). Participants tried harder to detect $\mathrm{T} 2$ and therefore succeeded more often. However, earlier research has shown that offering performance-related monetary rewards does not improve attentional-blink performance (Olivers \& Nieuwenhuis, 2005). Therefore, the results can probably be better understood as resulting from a change in response criterion ( $\beta$ in signal-detection theory). That is, even in T2-present trials that participants would classify as low or even zero subjective visibility (i.e., below the $50 \%$-visibility criterion), participants generally have minimal conscious access to T2-but sufficient to justify a wager on T2 presence (i.e., above the $€ 0$ criterion $)^{1}$. This possibility is consistent with previous claims that post-decision wagering might avoid participants' reluctance to report weakly perceived stimuli (which biases introspective reports). Also, in Experiment 3, we will report some evidence consistent with the notion that participants fail to be complete in their reports about what they consciously saw.

But why is the rating pattern so extreme? That is, why did participants go for the highest wager on such a large proportion of the T2-present trials? One possibility is that participants realized that the economically optimal strategy for wagering in experiments like the current experiment is to wager high regardless of the weight of the sensory evidence (Clifford et al., 2007). Or participants may not have been

\footnotetext{
${ }^{1}$ Because participants cannot have minimal conscious access to $\mathrm{T} 2$ in T2-absent trials, the hypothesized criterion shift need not necessarily lead to 'T2 present' wagers on T2-absent trials (in Experiment 2) that would have been rated as $0 \%$ visibility in Experiment 1 . This might explain why, in T2-present trials, more trials move from the left end (in Experiment 1) to the right end of the scale (in Experiment 2) than in T2-absent trials.
}

aware of the optimal strategy, and instead wagered high because of a risk-seeking bias, a personality trait that can affect post-decision wagering (Fleming \& Dolan, 2010; Schurger \& Sher, 2008). Although we cannot exclude these possibilities, we believe they are unlikely, first, because the 'T2 absent' ratings in T2-absent trials were much more spread across the (left end of the) scale (i.e., which is economically suboptimal), second, because previous postdecision wagering studies have shown that people do not tend to wager optimally (Schurger \& Sher, 2008; Shields, Smith, Guttmannova, \& Washburn, 2005), and third, because other participants from the same population used the whole post-decision wagering scale in Experiment 4.

In any case, these data are important for two reasons. At the methodological level, the experiment (in comparison with Experiment 1) indicates a striking dissociation between two different measures of consciousness, which may contribute to the ongoing debate about the relative usefulness of these measures. With regard to consciousness during the attentional blink, the experiment suggests that Sergent and Dehaene's (2004) findings of bimodal perception ratings (largely replicated in Experiment 1) may be specific to a particular consciousness measure (subjective visibility) and, at least in this particular context, do not generalize to another consciousness measure (wagering). However, because the wager ratings in Experiment 2 showed no reliable attentional blink, the data do not directly address the question whether consciousness during the attentional blink is a gradual or all-or-none dimension.

\section{Experiment 3}

In Experiment 3, we combined subjective visibility ratings with a character-identification task that is more representative of attentional-blink research and that is more difficult than the word-detection task used by Sergent and Dehaene (2004). 
Method

All methods were identical to Experiment 1, except when noted below. Fourteen participants were asked to identify both T1 and T2 in an RSVP sequence. In this experiment, T1 (again colored cyan) and T2 were digits between 2 and 9. Distractors consisted of single random letters, except I, $\mathrm{O}, \mathrm{Q}$, and $\mathrm{S}$. The visual angle of the RSVP items was $1^{\circ} \times$ $1^{\circ}$. Again, T2 was absent in half of the trials, and $20 \%$ of all distractors were replaced by a blank screen. After the RSVP sequence, participants were asked to rate the visibility of $\mathrm{T} 2$ on the visibility scale, and then identify $\mathrm{T} 1$ and $\mathrm{T} 2$ by pressing the corresponding numeral keys on the keyboard. Participants were instructed to press a random numeral key to indicate a fully unperceived (and possibly absent) T2 . Like in Experiment 1, the visibility ratings occurred immediately after the RSVP stream (and before the identification of $\mathrm{T} 1$ and $\mathrm{T} 2$ ) to rule out the possibility that participants would forget information on T2 visibility (Sergent \& Dehaene, 2004).

\section{Results and discussion}

Trials in which $\mathrm{T} 1$ and $\mathrm{T} 2$ were correctly identified but in the wrong order were treated as correct. Trials in which T1 was not correctly identified were discarded $(M=15.1 \%)$. Mean visibility ratings (Fig. 1), $F(5,65)=21.7, p<.001$, and $\mathrm{T} 2$ identification accuracy (Fig. 4), $F(5,65)=9.0, p<.001$, in T2-present trials showed significant effects of lag, indicating a typical attentional blink.

Figure 2 shows that the distributions of visibility ratings in T2-absent trials were similar to those in Experiment 1, with a single peak at $0 \%$ visibility ( $>60 \%$ of the trials). Crucially, the distributions for T2-present trials showed a very different pattern than in Experiment 1: instead of bimodal distributions, the visibility ratings showed rather uniform distributions. Furthermore, the attentional blink was characterized by a gradual displacement of subjective ratings toward lower subjective degrees of visibility, as evidenced by a significant lag $\times$ visibility interaction, $F(30,390)=5.8, p<.001$, a pattern consistent with the notion of a gradual transition between nonconscious and conscious perception.

As in the previous experiments, we conducted a linear regression analysis of the grand-average rating distributions for T2-present trials (lags 1-6). The present predictor (except at lag $6, p=.001$ ) and the absent predictor did not significantly contribute to these regressions, suggesting that contrary to what was found in Sergent and Dehaene (2004), and to a smaller extent in Experiment 1, participants did not use the subjective visibility scale in an all-or-none fashion. We conducted similar linear regression analyses of the rating distribu-

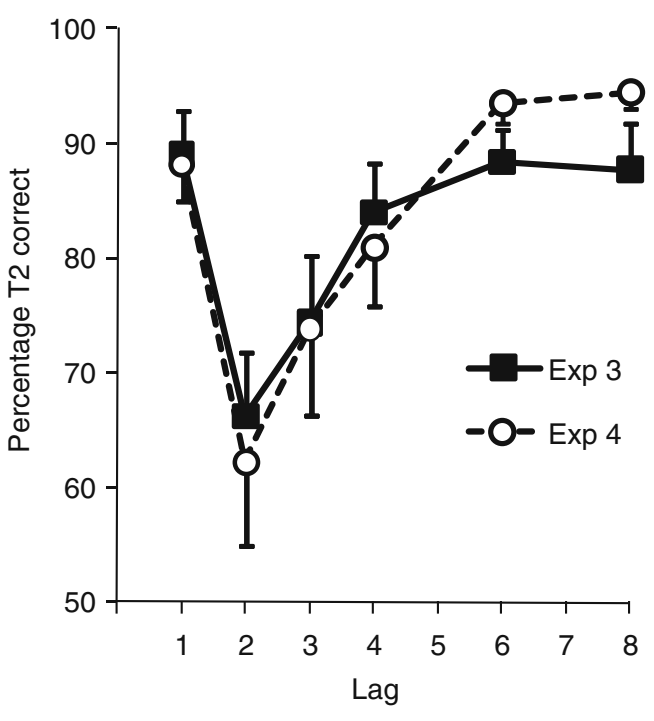

Fig. 4 Average T2 identification accuracy (given correct identification of T1) as a function of T1-T2 lag in Experiments 3 and 4. Error bars reflect standard errors of the mean

tions of individual participants, focusing specifically at lag 2 , where the attentional blink was most pronounced. These analyses confirmed the analysis of the grand-average rating distributions: there was only 1 out of 14 participants for which both the present and absent predictor significantly contributed to the regression. Visual inspection confirmed that this was the only participant with distinct peaks at 0 and $100 \%$ visibility.

Proponents of the post-decision wagering measure have suggested that participants are sometimes reluctant to report weakly perceived stimuli in introspective reports like subjective visibility ratings. To address this possibility, we examined whether $\mathrm{T} 2$ identification performance was above chance level (12.5\%) in trials during the attentional blink (lags 2 and 3) in which participants claimed to have not seen T2 ( $0 \%$ visibility) and guessed its identity. Because some participants had few $0 \%$ visibility trials, we pooled the 129 trials of all 14 participants. Consistent with the notion of incomplete report, T2 identification performance for these trials was $24.8 \%$. However, it is important to note that these data are equally consistent with the possibility that the abovechance task performance reflects nonconscious knowledge (by the guessing criterion; Dienes, 2008).

\section{Experiment 4}

In Experiment 4, we combined the more difficult identification task used in Experiment 3 with the post-decision wagering method used in Experiment 2. Twelve students took part in the experiment. 
Results and discussion

Trials in which $\mathrm{T} 1$ and $\mathrm{T} 2$ were correctly identified but in the wrong order were treated as correct. Trials with an incorrect $\mathrm{T} 1$ identification were discarded $(M=11.1 \%)$. Mean wager scores (Fig. 1), $F(5,55)=19.0, p<.001$, and T2 identification accuracy (Fig. 4), $F(5,55)=11.3, p=$ .001 , in T2-present trials showed significant effects of lag, indicating a typical attentional blink.

The wager distributions, plotted in Fig. 2, show a slightly complicated pattern. Almost all T2-absent trials were identified correctly ( $\sim 85 \%$ wagers on ' $\mathrm{T} 2$ absent'); the wagers were spread across the left side of the scale, with a small peak at $€ 0.60$ and a pronounced peak at $€ 0.20$, suggesting that participants were often uncertain of their decision. The wagers on T2-present trials showed a somewhat similar spread across the right side of the scale, with distinct peaks at $€ 0.20$ and $€ 0.60$. Importantly, during the attentional-blink period the wagers gradually shifted towards lower values, as evidenced by a significant lag $\times$ visibility interaction, $F(30,330)=12.2, p<.001$, such that at lag 2 the wagers were fairly well spread across the whole scale. There is a hint of a discontinuity between $€ 0.60$ and $€ 0.20$ ('T2 absent'), but nonlinear bifurcation models predict such a discontinuity immediately below the perceptual threshold (i.e., to the left of $€ 0$ ), not halfway the T2-absent scale (see Sergent \& Dehaene, 2004, Fig. 1). A linear regression analysis of the grand-average wager distributions for T2-present trials (lags 1-6) indicated a significant contribution of the present predictor for lags $4(p=.01)$ and $6(p<.001)$, but no contribution (and even negative beta coefficients) for the absent predictor. This was corroborated by regression analyses of the individual wager distributions for lag 2, which revealed only one participant (with distinct peaks at $€ 20$ for T2 'absent' and 'present') with significant contributions of both the absent and present predictors. Thus, the wager distributions in Experiment 4 largely converge with the subjective visibility distributions in Experiment 3 in suggesting that during the attentional blink perceptual target representations are progressively degraded.

Although Fig. 2 shows marked differences between the distributions in Experiments 3 and 4, they are not as striking as the differences between Experiments 1 and 2 and do not suggest a substantial criterion shift; that is, there is a fairly good correspondence between the percentages of $0 \%$ visibility trials in Experiment 3 and the percentages of ' $\mathrm{T} 2$ absent' wagers in Experiment 4.

\section{General discussion}

The attentional blink is an attractive paradigm for consciousness research because stimuli that are physically identical are sometimes consciously perceived and sometimes not. Sergent and Dehaene (2004) exploited this property to examine whether the transition between nonconscious and conscious perception is gradual or discontinuous. The participants in their experiment, when asked to rate the subjective visibility of targets on a 21 point scale, showed an extreme rating pattern: during the attentional blink T2s were either detected as well as targets outside the attentional-blink period or not detected at all.

In the 4 experiments reported here, we found mixed evidence for such all-or-none perception. In Experiment 1, we adopted Sergent and Dehaene's (2004, Experiment 1) methods, but using a 7-point instead of 21-point rating scale. The subjective visibility ratings obtained in this experiment were bimodally distributed, but the ' $\mathrm{T} 2$ seen' modus was not peaked but rather spread out, much like in Sergent et al. (2005). This may, in part, reflect the highpass filter function of a coarser scale, because when we regrouped Sergent and Dehaene's 21-bin data into 7 bins, the distribution of ratings across these bins looked remarkably similar to our data in Experiment 1 . However, the data are also consistent with the hypothesis (cf. Overgaard et al., 2006) that the disproportional use of the extreme ratings ( 0 and $100 \%$ visibility) in Sergent and Dehaene's (2004) experiment is in part due to the fact that the intermediate points on their 21-point scale did not have sufficient meaning to the participants. Nonetheless, the regression analyses of our (more spread-out) 7-point scale ratings replicated Sergent and Dehaene's (2004) finding that the visibility ratings in attentional-blink trials can be described as a bimodal mixture of seen (all) and not-seen (none) perceptual states.

Whereas in Experiment 1 we used Sergent and Dehaene's (2004) word-detection task, in Experiments 3 and 4 we used a more difficult and commonly used character-identification task. The visibility ratings in Experiment 3 were broadly (not bimodally) distributed, and the attentional blink was characterized by a gradual displacement toward lower subjective visibility ratings, consistent with continuous-transition models. It is possible that subjects have graded access to stimulus information at some levels of representation (e.g., the characters in Experiment 3) while having all-or-none access to other levels of stimulus representation (e.g., word form and meaning in Experiment 1). Even if subjects initially have graded conscious access to word form and meaning (e.g., because the $\mathrm{T} 2+1$ distractor masks the target word), the process of interactive activation between representations at the letter level and the word level may lead to the automatic filling-in of missing information, resulting in a complete (i.e., "all") representation of the word (McClelland \& Rumelhart, 1981). Indeed, this interactive activation mechanism bears resemblance to the type of global reverberation that causes complete conscious access in 
workspace models of consciousness (e.g., Dehaene et al., 2003). Thus, it is possible that the type of all-or-none rating pattern obtained with Sergent and Dehaene's word-detection task is specific for words and other stimuli promoting interactive activation.

In Experiment 4, we combined the character-identification task with post-decision wagering and found a similar gradual displacement in wager scores. To the extent that postdecision wagering measures access consciousness (i.e., the degree to which information is accessible for verbal report, reasoning or action), these results provide additional support for continuous-transition models of (non)conscious perception. Intuitively, it seems that wagers are made highly deliberately and that post-decision wagering can be treated as a test of awareness of knowing. However, it is not trivial that access consciousness can be inferred from successful wagering, because subjects may be able to learn to gamble implicitly on the basis of nonconscious information. For example, gambling behavior may be driven by nonconscious, graded and probabilistic representations of evidence or value, even when people's conscious states reflect only discrete samples from these probability distributions (Vul, Hanus, \& Kanwisher, 2009). Furthermore, although it seems reasonable to assume that increased gradations of consciousness go hand in hand with higher wagers, wagering is susceptible to psychological factors irrelevant to access consciousness, such as risk aversion (Fleming \& Dolan, 2010; Schurger \& Sher, 2008), that are hard to control. Thus, although the results of Experiment 4 are consistent with discontinuous models, the strength of this evidence depends on the status of postdecision wagering as an effective method for measuring access consciousness. This is a topic of ongoing debate and research (e.g., Dienes \& Seth, 2010; Overgaard, Timmermans, Sandberg, Cleeremans, 2010; Persaud \& McLeod, 2008; Seth, 2008). For example, Sandberg et al. (in press) found in a visual masking experiment that postdecision wagering was a less sensitive and exhaustive measure of consciousness than the Perceptual Awareness Scale, a subjective visibility scale. The salient difference between post-decision wagering and introspective measures like subjective visibility is exemplified by the results of Experiment 2 in which the discontinuous rating pattern obtained in Experiment 1 disappeared altogether; indeed, the wager scores did not show an attentional blink (i.e., all T2s were 'seen').

Is it possible that subjects' perception during the attentional blink is always all-or-none and that our methods in Experiments 3 and 4 failed to capture this property? Individual subjects may show all-or-none (or bimodal) rating patterns that are invisible in the grand-average rating distributions due to "smearing" across subjects. However, this is ruled out by the results of the regression analyses for individual participants in Experiments 3 and 4, which showed that there were only two participants whose rating distributions during the attentional blink could be modeled as a mixture of 'seen' and 'not seen' states. It is harder to exclude the possibility that the perception of any given participant in Experiments 3 and 4 is characterized by a discontinuous threshold that changes position and therefore smears out across trials, causing a seemingly continuous rating distribution. But if this is the case, one would expect a similar variability in threshold in the word-detection task of Experiment 1 and Sergent and Dehaene (2004) - a possibility that seems inconsistent with the clearly bimodal rating distributions in these experiments.

Alternatively, is it possible that there is always a continuous transition between nonconscious and conscious perception during the attentional blink, and that Sergent and Dehaene's (2004) particular combination of methods failed to capture this continuous transition? This possibility is challenged by Experiments 2 and 3 of Sergent and Dehaene, which showed that participants - with the same instructions as in Experiment 1 -used the subjective visibility scale in a continuous fashion when judging the visibility of masked words of variable durations (see also Sandberg et al., in press). Then again, it is possible that the discrepancy between Sergent and Dehaene's findings in the attentional-blink task and masking task reflects a response bias: participants, at least under Sergent and Dehaene's experimental conditions, may tend to use the subjective visibility ratings in an all-or-none fashion when they know objective visibility of $\mathrm{T} 2$ is all-or-none (attentional blink), and in a more graded fashion when they know objective visibility of the target changes from trial to trial (masking). Future research that compares consciousness ratings across different types of paradigms should take into account this type of response bias.

Taken together, our results cast doubt on Sergent and Dehaene's (2004) proposal that perception during the attentional blink has an all-or-none character. Evidence for a discontinuous transition between nonconscious and conscious perception can be obtained under specific conditions (e.g., when detecting words). However, our results show that under different conditions (e.g., character discrimination, a task more commonly used in attentionalblink research), evidence obtained with two different consciousness measures favors a continuous transition between nonconscious and conscious perception during the attentional blink.

Author Note The authors thank Heleen Slagter for providing the EPrime script of Sergent and Dehaene's attentional-blink task, and Claire Sergent, Stanislas Dehaene and Brad Wyble for helpful comments on an earlier version of this manuscript. This research was supported by a VIDI-grant from the Netherlands Organization for Scientific Research. 
Open Access This article is distributed under the terms of the Creative Commons Attribution Noncommercial License which permits any noncommercial use, distribution, and reproduction in any medium, provided the original author(s) and source are credited.

\section{References}

Clifford, C. W. G., Arabzadeh, E., \& Harris, J. A. (2007). Getting technical about awareness. Trends in Cognitive Sciences, 12, 54-58.

Dehaene, S., Naccache, L., Cohen, L., Bihan, D. L., Mangin, J. F., Poline, J. B., \& Riviere, D. (2001). Cerebral mechanisms of word masking and unconscious repetition priming. Nature Neuroscience, 4, 752-758.

Dehaene, S., Sergent, C., \& Changeux, J. P. (2003). A neuronal network model linking subjective reports and objective physiological data during conscious perception. Proceedings of the National Academy of Science, 100, 8520-8525.

Dienes, Z. (2008). Subjective measures of unconscious knowledge. Progress in Brain Research, 168, 49-64.

Dienes, Z., \& Seth, A. (2010). Gambling on the unconscious: A comparison of wagering and confidence ratings as measures of awareness in an artificial grammar task. Consciousness and Cognition, 19, 674-681.

Fleming, S. M., \& Dolan, R. J. (2010). Effects of loss aversion on post-decision wagering: Implications for measures of awareness. Consciousness and Cognition, 19, 352-363.

Koch, C., \& Preuschoff, K. (2007). Betting the house on consciousness. Nature Neuroscience, 10, 140-141.

Lamme, V. A. (2003). Why visual attention and awareness are different. Trends in Cognitive Sciences, 7, 12-18.

Lamme, V. A., Supèr, H., Landman, R., Roelfsema, P. R., \& Spekreijse, H. (2000). The role of primary visual cortex (V1) in visual awareness. Vision Research, 40, 1507-1521.

Martens, S., \& Wyble, B. (2010). The attentional blink: Past, present, and future of a blind spot in perceptual awareness. Neuroscience and Biobehavioral Reviews, 34, 947-957.

McClelland, J. L., \& Rumelhart, D. E. (1981). An interactive activation model of context effects in letter perception: I. An account of basic findings. Psychological Review, 88, 375-407.
Moutoussis, K., \& Zeki, S. (2002). The relationship between cortical activation and perception investigated with invisible stimuli. Proceedings of the National Academy of Science, 99, 9527-9532.

Olivers, C. N. L., \& Nieuwenhuis, S. (2005). The beneficial effect of concurrent task-irrelevant mental activity on temporal attention. Psychological Science, 16, 265-269.

Overgaard, M., Rote, J., Mouridsen, K., \& Ramsøy, T. Z. (2006). Is conscious perception gradual or dichotomous? A comparison of report methodologies during a visual task. Consciousness and Cognition, 15, 700-708.

Overgaard, M., Timmermans, B., Sandberg, K., \& Cleeremans, A. (2010). Optimizing subjective measures of consciousness. Consciousness and Cognition, 19, 682-684.

Persaud, N., \& McLeod, P. (2008). Wagering demonstrates subconscious processing in a binary exclusion task. Consciousness and Cognition, 17, 565-575.

Persaud, N., McLeod, P., \& Cowey, A. (2007). Post-decision wagering objectively measures awareness. Nature Neuroscience, 10, 257261.

Ramsøy, T. Z., \& Overgaard, M. (2004). Introspection and subliminal perception. Phenomenology and the Cognitive Sciences, 3, 1-23.

Sandberg, K., Timmermans, B., Overgaard, M., \& Cleeremans, A. (in press). Measuring consciousness: Is one measure better than the other? Consciousness and Cognition.

Schurger, A., \& Sher, S. (2008). Awareness, loss aversion, and postdecision wagering. Trends in Cognitive Sciences, 12, 209-210.

Sergent, C., Baillet, S., \& Dehaene, S. (2005). Timing of the brain events underlying access to consciousness during the attentional blink. Nature Neuroscience, 8, 1391-1400.

Sergent, C., \& Dehaene, S. (2004). Is consciousness a gradual phenomenon? Evidence for an all-or-none bifurcation during the attentional blink. Psychological Science, 15, 720-728.

Seth, A. K. (2008). Post-decision wagering measures metacognitive content, not sensory consciousness. Consciousness and Cognition, 17, 981-983.

Shields, W. E., Smith, D. J., Guttmannova, K., \& Washburn, D. A. (2005). Confidence judgments by humans and Rhesus monkeys. The Journal of General Psychology, 213, 165-186.

Vul, E., Hanus, D., \& Kanwisher, N. (2009). Attention as inference: Selection is probabilistic; responses are all-or-none samples. Journal of Experimental Psychology: General, 138, 546-560. 